\title{
A novel baculovirus-derived promoter with high activity in the Baculovirus Expression System
}

\author{
María Martínez-Solís ${ }^{1,2}$, Silvia Gómez-Sebastián ${ }^{3}$, José M Escribano ${ }^{4}$, Agata K Jakubowska ${ }^{1}$, Salvador \\ Herrero Corresp. 1,2 \\ 1 Department of Genetics, Universitat de València, Burjassot, Spain \\ 2 Estructura de Recerca Interdisciplinar en Biotecnologia i Biomedicina (ERI BIOTECMED), Universitat de València, Burjassot, Valencia, Spain \\ 3 Alternative Gene Expression S.L. (ALGENEX), Madrid, Spain \\ 4 Departamento de Biotecnología, Instituto Nacional de Investigación y Tecnología Agraria y Alimentaria (INIA), Madrid, Spain \\ Corresponding Author: Salvador Herrero \\ Email address: sherrero@uv.es
}

The baculovirus expression vector system (BEVS) has been widely used to produce a large number of recombinant proteins, and is becoming one of the most powerful, robust, and cost-effective systems for the production of eukaryotic proteins. Nevertheless, as in any other protein expression system, it is important to improve the production capabilities of this vector. The orf46 viral gene was identified among the most highly abundant sequences in the transcriptome of Spodoptera exigua larvae infected with its native baculovirus, the $S$. exigua multiple nucleopolyhedrovirus (SeMNPV). Different sequences upstream of the orf46 gene were cloned, and their promoter activities were tested by the expression of the GFP reporter gene using the Autographa californica nucleopolyhedrovirus (AcMNPV) vector system in different insect cell lines (Sf21, Se301, and Hi5) and in larvae from S. exigua and Trichoplusia ni. The strongest promoter activity was defined by a 120 nt sequence upstream of the ATG start codon for the orf46 gene. On average, GFP expression under this new promoter was more than two fold higher than the expression obtained with the standard polyhedrin (polh) promoter. Additionally, the orf46 promoter was also tested in combination with the pph promoter, revealing an additive effect over the polh promoter activity. In conclusion, this new characterized promoter represents an excellent alternative to the most commonly used baculovirus promoters for the efficient expression of recombinant proteins using the BEVS. 


\section{A novel baculovirus-derived promoter with high activity in 2 the Baculovirus Expression System}

3 María Martínez-Solís ${ }^{1,2}$; Silvia Gómez-Sebastián ${ }^{3}$; José M. Escribano ${ }^{4}$; Agata K. Jakubowska ${ }^{1}$;

4 Salvador Herrero ${ }^{1,2}$

$5 \quad{ }^{1}$ Department of Genetics, Universitat de València, Burjassot, Spain

$6{ }^{2}$ Estructura de Recerca Interdisciplinar en Biotecnologia i Biomedicina (ERI BIOTECMED),

7 Universitat de València, 46100-Burjassot, Valencia, Spain

$8 \quad{ }^{3}$ Alternative Gene Expression S.L. (ALGENEX), 28223 Madrid, Spain

$9{ }^{4}$ Departamento de Biotecnología, Instituto Nacional de Investigación y Tecnología Agraria y

10 Alimentaria (INIA), 28040 Madrid, Spain

12 Corresponding author:

13 Salvador Herrero

14 Dr. Moliner 50, Burjassot, Valencia, 46100, Spain

15 Email address: sherrero@uv.es 


\section{Abstract}

17 The baculovirus expression vector system (BEVS) has been widely used to produce a large 18 number of recombinant proteins, and is becoming one of the most powerful, robust, and cost19 effective systems for the production of eukaryotic proteins. Nevertheless, as in any other protein 20 expression system, it is important to improve the production capabilities of this vector. The orf46 21 viral gene was identified among the most highly abundant sequences in the transcriptome of 22 Spodoptera exigua larvae infected with its native baculovirus, the $S$. exigua multiple 23 nucleopolyhedrovirus (SeMNPV). Different sequences upstream of the orf46 gene were cloned, 24 and their promoter activities were tested by the expression of the GFP reporter gene using the 25 Autographa californica nucleopolyhedrovirus (AcMNPV) vector system in different insect cell 26 lines (Sf21, Se301, and Hi5) and in larvae from S. exigua and Trichoplusia ni. The strongest 27 promoter activity was defined by a $120 \mathrm{nt}$ sequence upstream of the ATG start codon for the 28 orf46 gene. On average, GFP expression under this new promoter was more than two fold higher 29 than the expression obtained with the standard polyhedrin (polh) promoter. Additionally, the 30 orf46 promoter was also tested in combination with the polh promoter, revealing an additive 31 effect over the polh promoter activity. In conclusion, this new characterized promoter represents 32 an excellent alternative to the most commonly used baculovirus promoters for the efficient 33 expression of recombinant proteins using the BEVS. 
35

36

37

38

39

40

41

42

43

44

45

46

47

48

49

50

51

52

53

54

55

56

57

58

59

60

61

62

63

64

\section{Introduction}

Baculoviruses are enveloped, double-stranded DNA viruses pathogenic to invertebrates, preferably Lepidoptera. Their specificity to kill a narrow spectrum of insects and their safety for humans, plants, and non-target insects, make them a good biological control agent. In addition, since 1983, baculoviruses have been extensively used as protein expression vectors in insect cells (Smith, Summers, and Fraser 1983). The baculovirus expression vector system (BEVS) has been widely used to produce a large number of recombinant proteins, and several systems using different strategies for the generation of the recombinant viruses have been developed ( $\mathrm{Li}$ et al. 2012; van Oers, Pijlman, and Vlak 2015). The high popularity reached by this system is due to its ability to produce large amounts of active proteins, together with its ability to introduce posttranslational modifications in the expressed protein, similar to mammalian cells, such as glycosylation or phosphorylation (O’Reilly, Miller, and Luckow 1994).

Similar to most viruses, the baculovirus gene expression has a temporal regulation which can be divided into 3 main phases: the early, late, and very late phases (Friesen 1997; Lu and Miller 1997; Jarvis 2009). The expression of the early genes does not require prior viral protein synthesis and precedes viral DNA replication. The late phase is a period for viral DNA replication, and the very late phase is characterized by the production of viral particles. In this final phase of infection the expression of the polyhedrin and p10 structural proteins predominate, and these comprise the major proportion of the cell protein mass. The high transcription yield of the promoters of these two proteins has been exploited in the BEVS to express foreign proteins (G. F. Rohrmann 1999). The baculovirus of Autographa californica (A. californica nucleopolyhedrovirus, AcMNPV) is the main viral species used as an expression vector for recombinant protein expression using the BEVS. The polyhedrin and the p10 promoters from AcMNPV have been extensively used for the expression of foreign proteins with this system. However, recombinant protein expression yields not only depend on the promoter used, but also on the host cell line, as well as the characteristics of the foreign gene (Morris and Miller 1992).

1 Several strategies have been developed to improve the production of functional proteins in insect 2 cells. For instance, modification of the expression vectors by the addition of DNA elements 63 involved in protein expression processes can enhance the production yields of recombinant 64 proteins (Lo et al. 2002; Venkaiah et al. 2004; Manohar et al. 2010; Tiwari et al. 2010; Gómez- 
65 Sebastián, López-Vidal, and Escribano 2014). Nevertheless, one of the main cis-regulatory

66 elements affecting the protein expression levels is the promoter. To date, different types of

67 promoters have been tested in the BEVS to improve recombinant protein expression. Viral

68 promoters such as vp39 or 39K, and promoters derived from insect larvae such as the hexamerin-

69 derived promoter pB2 from Trichoplusia ni (López-Vidal et al. 2013) showed high levels of

70

71

72

73

74

75

76

77

78

79

80

81

82

83

84

85

86

87

88

89

90

91

92

93

expression of recombinant proteins. In other cases, the combination of some of these promoters with the conventional promoters exhibited higher expression levels of the recombinant proteins than the standard late promoters alone (Thiem and Miller 1990; Morris and Miller 1992; Ishiyama and Ikeda 2010; Lin and Jarvis 2012).

In a previous work, the transcriptional pattern of the Spodoptera exigua multiple nucleopolyhedrovirus (SeMNPV) during the infective process in its natural host revealed very high levels of expression for the orf46 viral gene (Pascual et al. 2012). Since the orf46 gene codes for the structural protein polyhedron envelope protein (PEP), we hypothesized that its expression could be regulated by a strong promoter. In this study, we have determined the core regulatory sequence for the gene (orf46) derived from the SeMNPV and we have examined its ability to drive the expression of recombinant proteins in insect cells using the BEVS. Different sequences upstream of the ATG start codon of the orf46 gene were cloned, and their promoter activities were tested by the expression of GFP as a reporter gene using the AcMNPV system in different insect cell lines. In addition, the promoter activity of this region was tested when combined with the standard polyhedrin promoter derived from the AcMNPV.

\section{Materials and Methods}

\section{Culture cells and insects}

The Spodoptera exigua (Se301) and Spodoptera frugiperda (Sf21) cell lines were cultured at 25 ${ }^{\circ} \mathrm{C}$ in Gibco ${ }^{\circledR}$ Grace's Medium (1X) (Life technologies ${ }^{\mathrm{TM}}$ ) supplemented with $10 \%$ heatinactivated fetal bovine serum (FBS). The Trichoplusia ni (High Five, Hi5) cell line was cultured at $27^{\circ} \mathrm{C}$ in TNMFH medium supplemented with $10 \% \mathrm{FBS}$ and gentamicin $(50 \mu \mathrm{g} / \mathrm{ml})$. S. exigua larvae were maintained in the laboratory, reared on an artificial diet at $25 \pm 3{ }^{\circ} \mathrm{C}$ with $70 \pm 5 \%$ relative humidity and a photoperiod of 16/8 hours (light/dark). Trichoplusia ni (cabbage looper) 
94 larvae were reared on an artificial insect diet and were kept in growth chambers at $22 \pm 1{ }^{\circ} \mathrm{C}$ 95 under controlled humidity (50\%) and light period ( $8 \mathrm{~h} /$ day $)$ conditions.

\section{Sequence identification}

97 The transcriptional regulatory region was determined by in silico analysis of the sequences 98 derived from the Roche 454 FLX and Sanger methods obtained from the transcriptome of $S$. 99 exigua larvae which included samples of SeMNPV-infected larvae (at the latest stage of the infection) (Pascual et al. 2012). First, the ten ORFs with the highest expression levels were obtained based on their maximum coverage. Then, the upstream region from the ATG start codon of orf46 was analyzed in silico and manually for the prediction of the transcriptional regulatory region. Using promoter prediction software (http://www.fruitfly.org/seq_tools/promoter.html), we identified a transcription start site and other motifs characteristic for baculovirus promoters. A sequence of $300 \mathrm{bp}$ upstream of the predicted start site was selected as an initial candidate region to act as a promoter.

\section{Construction of recombinant baculoviruses}

108

109

110

111

112

113

114

115

116

117

118

119

120

121

Several baculovirus-transfer plasmids containing different fragments of the 5' region of the orf46 gene driving the expression of GFP were generated using the AcMNPV vector system (Fig. 1). The GFP gene was initially cloned under the control of the polyhedrin promoter (polh) to generate the pFB-PL-GFP vector (López-Vidal et al. 2013) (from now, polh-GFP). The initial pSeL and pSeS promoter sequences were obtained by PCR amplification using SeMNPV genomic DNA as template. PCR amplifications were performed using specific primers which added Bstz17I and SpeI restriction sites. The polh promoter was then replaced by the pSeL or pSeS fragments into the Bstz17I and SpeI sites, to generate the pSeL-GFP and pSeS-GFP vectors, respectively. The pSeL140 and pSeL120 sequences were amplified by PCR from the pSeL-GFP vector using two specific primers. The first primer included the corresponding 5 ' region of $\mathrm{pSeL}$ and a $B s t Z 17 \mathrm{I}$ restriction site, and the second primer was designed to amplify from a 3' region of the GFP gene containing an AvrII restriction site. These sequences were cloned into the BstZ17I and AvrII sites of the polh-GFP vector by replacement of the polh promoter, generating the pSeL140-GFP and pSeL120-GFP vectors. 
122 The vector combining two promoters (polh-pSeL-GFP) was constructed by modification of the 123 polh-GFP vector. The pSeL120 promoter fragment was obtained by PCR using specific primers 124 which added XhoI and $A v r \mathrm{II}$ restriction sites. The resulting fragment was inserted into the XhoI 125 and $A v r$ II sites of the polh-GFP vector, generating the polh-pSeL-GFP vector containing both of 126 the polh and pSeL120 promoters in tandem. Additionally, the DNA sequence corresponding to 127 the p131 (homolog to orf46 in AcMNPV) promoter was chemically synthesized (GenScript) and 128 flanked by BstZ17I and SpeI restriction sites. This was cloned into a pFB vector to control the 129 expression of the GFP gene, generating the p131-GFP vector. Figure 1 shows a schematic 130 representation of all of the different recombinant baculoviruses generated in the present work.

131

132

133

134

135

136

137

138

139

140

141

142

143

144

145

146

148

149

150

147 Infection assays in culture cells and insects

The sequences of the primers employed for the cloning of the different constructs are summarized at table S1.

The recombinant baculoviruses were obtained using the Bac-To-Bac ${ }^{\circ}$ baculovirus expression system (Invitrogen, USA) following the manufacturer's instructions. Plasmids generated in the previous step were used to transform E. coli $\mathrm{DH} 10 \mathrm{Bac}^{\mathrm{TM}}$ heat-shock competent cells and generate the corresponding recombinant bacmids. Bacmids were purified and used to transfect Sf21 cells using Cellfectin ${ }^{\circledR}$ II Reagent (Invitrogen) following the manufacturer's instructions. The resulting baculoviruses were collected after 4-5 days of incubation at $27^{\circ} \mathrm{C}$. These baculoviruses were amplified once to obtain high-titer stocks for further experiments, and the viral titers were determined by quantitative PCR (qPCR) using specific primers (Table S1). For that purpose, viral DNAs were treated using Prepman reagent (Applied Biosystems) following the manufacturer's instructions and were quantified by comparing the obtained $\mathrm{Ct}$ values against a standard curve of known viral concentration. Viral titers used in the standard curve were obtained by end point dilution, a method that does not consider non-infective viruses. The viral titers were expressed as baculoviruses per milliliter (BVs/ml).

48 Cells (Se301, Sf21, and Hi5) were cultured in 24-well plates at a confluence of 70\%, then the cells were infected with the different recombinant baculoviruses at a multiplicity of infection (MOI) of 5. The cells were collected at different times post-infection by low speed centrifugation 
151 (3000 rpm, $5 \mathrm{~min}$ ) to avoid cell lysis, and kept at $-20{ }^{\circ} \mathrm{C}$ until the quantification of GFP 152 expression. Last instar S. exigua and $T$. $n i$ larvae were injected with $5 \mu 1$ of recombinant 153 baculoviruses containing $5 \times 10^{4} \mathrm{BVs}$. Larvae were maintained at $25^{\circ} \mathrm{C}$ and $28{ }^{\circ} \mathrm{C}$, respectively, 154 and after 72 hours post-infection (hpi) were frozen at $-20^{\circ} \mathrm{C}$ until they were processed for GFP 155 quantification.

156

157 158

159

160

161

162

163

164

165

166

167

168

169

170

171

172

173

174

175

176

177

178

\section{Analysis of GFP expression}

Frozen cells from the infection assays were resuspended in a lysis buffer $(50 \mathrm{mM}$ Tris- $\mathrm{HCl} \mathrm{pH}$ $7.5,100 \mathrm{mM} \mathrm{NaCl}, 1 \mathrm{mM} \mathrm{DTT}, 5 \%$ glycerol), incubated for $5 \mathrm{~min}$ at room temperature, and centrifugated at $16,000 \mathrm{xg}$ for $1 \mathrm{~min}$. The supernatant was collected to measure GFP expression by fluorescence in a microplate reader (Infinite ${ }^{\circledR} 200$ PRO NanoQuant, TECAN) (excitation 485 $\mathrm{nm}$, emission $535 \mathrm{~nm}$ ). Each value was obtained by measuring each sample 4 times.

Occasionally, the production of GFP was confirmed by direct observation of the GFP protein band in SDS-PAGE, suggesting a good correlation between GFP intensity and protein abundance. Frozen larvae were homogenized in $1 \mathrm{ml}$ of extraction buffer $(0.01 \%$ de Triton X100, $1 \mathrm{mM}$ de PMSF, and DTT $25 \mathrm{mM}$ in PBS 1x). Homogenates were centrifuged at $1800 \mathrm{xg}$ for $30 \mathrm{~min}$ at $4{ }^{\circ} \mathrm{C}$, and the supernatant was collected to measure GFP as described above. The values correspond to at least two independent replicates for all of the experiments. Statistical analyses were performed by Dunnett's Multiple Comparison Test using the GraphPad Prism program (GraphPad software Inc., San Diego, CA, USA).

\section{Results}

\section{Expression of viral genes and promoter selection}

Expression levels of the SeMNPV genes were monitored by mapping of the viral reads on the transcriptome of S. exigua infected larvae (Pascual et al., 2012). As expected, the most abundant reads were mapping on the orfl which corresponded to the polyhedrin gene (Table 1). The second most abundant ORF mapped, corresponded to the orf 46 gene of SeMNPV. Orf46 codes for the polyhedron envelope protein (PEP), a structural protein that surrounds the polyhedra of the viral particles. In addition, other genes highly expressed during the infection were orf 127 and 
179 orf122. Given the high expression observed for the orf 46 gene under our experimental 180 conditions, and its role as a structural protein, we decided to explore the possibility of using its regulatory sequence as a promoter for foreign gene expression using the BEVS.

182 Detailed analysis of the 454-derived reads mapping to the SeMNPV genome predicted the 183 transcription start site (site +1 ) of orf46 at position 89150 (which referred to the reverse complementary SeMNPV genome, GenBank acc: AF169823.1). A region of $301 \mathrm{nt}$ upstream of 185 the start codon of orf46 from SeMNPV was initially selected as the promoter sequence. The in 186 silico analysis predicted a promoter between nucleotides 224-269 from the selected sequence that revealed the presence of a TAAG motif. This TAAG motif was in an AT rich region, and it was described as a typical transcriptional initiation site of late and very late baculovirus promoters (Lu and Miller 1997).

\section{Orf46 promoter activity in insect cells}

191

192

193

194

195

196

197

198

199

200

201

202

203

204

205

206

207

To determine the promoter activity of the region upstream of the orf 46 gene from SeMNPV and its homologous equivalent region in AcMNPV (p131), different constructs were obtained and tested for their ability to drive the expression of the GFP reporter gene (Figs. 2 and 3) using the BEVS. Se301, Sf21, and Hi5 cells were infected with recombinant AcMNPV baculoviruses expressing GFP under the different promoter regions, and their activities were compared to the activity obtained with the standard polyhedrin (polh) promoter from AcMNPV. The GFP expression yields obtained for the $300 \mathrm{bp}$ fragment upstream of orf 46 (pSeL) was equivalent to that obtained with the polh promoter in the Se301 and Sf21 cells. Interestingly, for the Hi5 cells, the GFP expression mediated by the pSeL promoter was about two-fold higher than that obtained using the polh promoter. The deletion of the 25 nucleotides in the 3' region of the pSeL sequence (pSeS) strongly affected its promoter activity in the three cell types tested, revealing the importance of this region in the activity of the orf46 promoter. The homolog promoter in AcMNPV (p131) showed a significantly lower expression level than $\mathrm{pSeL}$ and the control polh in all of the cell lines tested (Fig. 2).

In order to further delimit the promoter region, two additional constructs containing 120 and 140 nucleotides upstream of the orf46 gene were also tested (pSeL120 and pSeL140, respectively). The reduction in the promoter size had a positive impact on the promoter activity in most cases, 
208 as the GFP expression was double that seen when compared to the polh promoter (Figs. 2 and 3).

209 The highest expression levels were observed for the region consisting of the $120 \mathrm{nt}$ upstream of

210 the orf46 gene. When compared to the polh promoter, pSeL120 showed an increase in expression

211 of more than two fold in all of the cell lines tested. These results strongly suggest that pSeL120

212 could be considered a useful promoter with the capacity to significantly increase the expression

213 yields obtained with the conventional polyhedrin promoter in the BEVS.

\section{Activity of the pSeL120 in combination with standard promoter in insect cells}

215 In a subsequent analysis, a recombinant baculovirus expressing GFP under the control of a 216 promoter combining the pSeL120 and polh in tandem (polh-pSeL) was generated and tested for 217 its expression levels. After infecting insect cells with this recombinant baculovirus, we observed 218 an additive effect over the two promoters used separately in different recombinant baculoviruses, 219 increasing the polh-pSeL promoter GFP expression to around 3-fold of the levels obtained with 220 the polh or pSeL120 promoters alone (Figs. 3 and 4). This additive effect was observed with 221 small variations in the three insect cell lines tested. These results revealed the potential of 222 pSeL120 to be combined with other promoters in order to produce increased amounts of 223 recombinant proteins in the BEVS.

224

225

226

227

228

229

230

231

232

233

234

235

236

\section{Activity of the new promoters in baculovirus-infected insect larvae}

Although BEVS is mainly used for protein production in insect cell cultures, they can also be used to efficiently produce recombinant proteins in a cost-effective manner by using Lepidoptera larvae. We tested the activity of several of the above described new promoters in larvae from two species of Lepidoptera, the specific host of the SeMNPV, S. exigua and T. $n i$, commonly used for protein production using AcMNPV-based vectors. Last instar of S. exigua and T. $n i$ larvae were infected by intrahemocelical injection with the recombinant baculoviruses expressing GFP under the control of every promoter tested. After $48 \mathrm{hpi}$, the protein production was estimated by measuring the GFP fluorescence of the larval extracts (Fig. 5). For all of the viruses tested in both insect species, the GFP production using pSeL or pSeL120 was equivalent to that obtained with a baculovirus expressing this protein under the control of the polyhedrin promoter. For the baculovirus comprised of both the polh and pSeL120 promoters, the expression was similar to the polh control promoter in $T$. $n i$ larvae, and slightly lower in S. exigua larvae. 


\section{Discussion}

239 Despite the wide use of the BEVS since the early 1980s (Smith, Summers, and Fraser 1983), the

240

241

242

243

244

245

246

247

248

249

250

251

252

253

254

255

256

257

258

259

260

261

262

263

264

265

266 system remains in terms of productivity very similar to the one originally developed. It is worth pointing out the need for research in the improvement of the productivity by different approaches, as was previously shown for other eukaryotic and prokaryotic production platforms. Several strategies have been attempted to increase the production yields by introducing modifications and improvements at different levels. Some of the improvements in the BEVS have been focused on the modification of viral promoters (Manohar et al. 2010), or the introduction of regulatory sequences (Sano et al. 2002; Tiwari et al. 2010; Ge et al. 2014; Gómez-Sebastián, López-Vidal, and Escribano 2014). Other strategies were based on the deletion of non-essential genes of the vector (Hitchman et al. 2010; Hitchman et al. 2011). One standard strategy is the search for promoters which are stronger than those commonly used, such as the p10 and polyhedrin (polh) promoters, or chimeras of them employed in laboratory and industrial production (Thiem and Miller 1990; Ishiyama and Ikeda 2010; Lin and Jarvis 2012; López-Vidal et al. 2013). However, often the efficiency of the promoter also depends on the regulatory sequences around them and the type of cellular lines in which they are acting (Matsuura et al. 1987; Morris and Miller 1992; C H Gross and Rohrmann 1993; Lo et al. 2002). Thus, the development of new promoters to implement the cost-efficient production of recombinant proteins and to provide alternatives to the traditional promoters, still remains of interest.

Viral genes coding for structural proteins are usually regulated by strong promoters, since they need to be highly translated to produce the viral particles. Thus, they are good candidates to explore in the improvement of the BEVS. The promoter studied in this work regulates the expression of the orf46 gene from SeMNPV, which codes for the calyx/polyhedron envelope protein (PEP). The polyhedron envelope is an electron-dense structure that forms a smooth, seamless surface that surrounds polyhedra. The function of calyx/PE is to seal the surface of polyhedra and to enhance their stability (George F. Rohrmann 2013). Homologs of the PEP are found in the genomes of all lepidopteran nucleopolyhedroviruses. The PEP is associated with p10 fibrillar structures, and both proteins appear to be important for the proper formation of the 
267 polyhedron envelope (van Lent et al. 1990; Russell, Pearson, and Rohrmann 1991; C. H. Gross, 268 Russell, and Rohrmann 1994; Lee et al. 1996). PEP from AcMNPV was shown to be associated 269 with BV but not with ODV. It is abundantly produced during the late phase of infection (Wang et 270 al. 2010).

271 In the present study, we have described a new viral promoter sequence derived from the gene 272 that codes for the structural PEP from SeMNPV, showing better performance than the polh 273 promoter in the BEVS in different cell lines. By testing different sequences upstream of the ATG 274 start codon from the orf46 gene driving the expression of GFP, we have limited the essential 275 promoter sequence. The sequence corresponding to the $120 \mathrm{nt}$ just before the ATG start codon 276 (pSeL120) showed the strongest promoter activity when it was functioning in cultured cell lines. 277 On average, the expression under the pSeL120 promoter was at least 2 times higher than the 278 maximum expression levels reached using the standard polh promoter. Other groups have 279 investigated the characterization of new promoters for increased expression yields. Lin \& Jarvis 280 (Lin and Jarvis 2012) showed that the delayed early 39K promoter from AcMNPV produced 4281 fold more SEAP protein than the polyhedrin promoter in Sf21 cells. López-Vidal et al. (López282 Vidal et al. 2013) isolated the pB2 promoter (promoter region of the Basic juvenile hormone283 suppressible protein 2, BJHSP-2) from the Lepidoptera T. $n i$ with activity in Sf21 cells. The pB2 284 promoter can drive the expression of GFP earlier in time, but it is not as strong as the polyhedrin 285 promoter. Ishiyama \& Ikeda (Ishiyama and Ikeda 2010) reported that the expression of GFP was 286 287 increased using the vp39 late promoter in comparison to the polyhedrin promoter in Bombix mori cultured cells.

288 Despite the high level of conservation and similarity between sequences from different virus 289 species, the homolog p131 sequence from AcMNPV showed the lowest promoter activity, even 290 lower than the control polh promoter. Such discrepancy could be explained by the fact that the 291 p131 transcription start site (predicted in silico) is not located in the TAAG region, and this 292 region seems to be very important in order to obtain high expression levels (as mentioned 293 above). Alternatively, it could also be possible that the activity of p131 in AcMNPV is not as 294 crucial as the orf46 activity in SeMNPV. This hypothesis is supported by some gene expression 295 data in AcMNPV in the literature. It has been published that the gene expression levels of pp34 296 (gene whose expression is controlled by p131 in AcMNPV) were considerably lower than the 
297 polyhedrin and p10 expression in infected Sf9 cells (Iwanaga et al. 2004). The analysis of the 298 transcriptome of AcMNPV-infected T. ni cells also showed lower expression levels of pp34 in 299 comparison to the polyhedrin and p10 genes (Chen et al. 2013).

300

An additional improvement with regard to the protein expression was obtained when the

301

302

303

304

305

306

307

308

309

310

311

312

313

314

315

316

317

318

319

320

321

322

323

324

325

326

pSeL120 promoter was combined with the polh promoter (polh-pSeL), resulting in increases of about 3-fold over the polh promoter and 1.5 fold over the pSeL120 promoter alone. Increases in protein production have also been reported by the combination of different promoters. Thiem \& Miller (Thiem and Miller 1990) showed that the combination of the vp39 and the polyhedrin promoter enhanced the expression of foreign genes compared to using those promoters alone in Sf cells, because this hybrid promoter showed regulation patterns of late and very late promoters. López-Vidal et al. (López-Vidal et al. 2013) also demonstrated an increase in GFP production of more than $20 \%$ at early times post-infection, and similar expression levels at very late times postinfection in Sf21 cells using a pB2-p10 promoter combination, with respect to conventional late promoters.

Although our results have shown a clear improvement of the pSeL promoter activity in different cell types, we could not observe such improvement when it was used for protein production in $S$. exigua and T. ni larvae. The difference in the promoter activity between the cell lines and larvae could be due to additional factors affecting the replication dynamics and/or promoter activity of the virus, as well as the timing selected for the processing of the larvae. Nevertheless, the pSeL120 promoter activity in larvae is equivalent to that obtained using the polh promoter, and no significant differences were observed, demonstrating that the promoter exhibits versatility and can be utilized in both cell lines (with high activity for a wide range of cell types) and insect larvae (with activity equal to the polh promoter).

When compared with homologous sequences in other viral species, we found a region of $50 \mathrm{nt}$ upstream of the ATG start codon that was highly conserved between them. Interestingly, removal of $25 \mathrm{nt}$ of this sequence downstream of the +1 start transcription site in mRNA abolishes the activity of the pSeS promoter. This observation suggests that this region is essential for the strong promoter activity as already proposed in previous studies. Weyer and Possee (Weyer and Possee 1988) showed that the 5'UTR regions are necessary for the maximum activity of the polyhedrin and p10 promoters. In agreement with that, expression levels for 
327 foreign proteins are related to the integrity of the 5'UTR region of the polyhedrin gene 328 (Matsuura et al. 1987; Luckow and Summers 1988). The sequence located between the TAAG 329 motif and the translation initiation site is known in baculoviruses as the burst sequence (BS) 330 (Weyer and Possee 1988). This is a sequence of about $50 \mathrm{nt}$ required for the efficient expression 331 of viral genes during the very late phase of infection. Studies of mutational analysis regarding the 332 BS region have demonstrated that BS are essential for efficient protein expression (Ooi, Rankin, 333 and Miller 1989; Weyer and Possee 1988), which agrees with our results. If we take into 334 consideration that most of those highly conserved 50 nucleotides are included in the 5'UTR of 335 the ORF46 transcript, it seems that the increase in expression found with the pSeL-derived 336 promoters is likely influenced by the effect of such sequences with respect to the access provided 337 to the RNApol, which affects the transcription and translation rates, and even increases mRNA 338 stability. The reason in this case is not known, but it has already been described that an upstream 339 sequence of the AcMNPV polyhedrin gene has an important function for mRNA transcription 340 and translation efficiencies (Min and Bishop 1991).

341 In conclusion, the sequence derived from the SeMNPV genome described in this work represents 342 a new promoter which is able to express, in most cases, higher yields of foreign proteins than the 343 polh promoter in the BEVS. Moreover, the combination of $\mathrm{pSeL}$ with the conventional polh 344 promoter showed higher activity for the expression of GFP than the pSeL or polh promoters 345 alone. Although additional validations of this promoter for the expression of recombinant 346 proteins other than GFP would be needed, these results represent a new improvement in the 347 production of recombinant proteins using the BEVS, with potential application in the cost348 efficient large-scale industrial production of biologics.

\section{Acknowledgments}

351 We want to thank Rosa Maria González-Martínez for her excellent help with insect rearing and laboratory management. 
353

354 355

356 357

\section{Figure legends}

Figure 1: Schematic summary of the recombinant baculoviruses carrying different promoter regions employed in this study.

(A) Nucleotide sequence upstream of the orf 46 gene from SeMNPV selected as a regulatory region (nucleotides 45417-47500 from AF169823). The arrows indicate the range of the fragments from the 5' to 3 ' sequence that were tested for promoter activity and the transcription initiation site $(+1)$. (B) Schematic representation of the recombinant baculoviruses generated which carry different fragments of the sequence upstream of the orf46 gene to test their promoter activity using GFP as a reporter. The white open arrows (polh) represent the polyhedrin promoter. The white boxes represent the GFP gene. The numbers indicate the first and the last nucleotides (from 5' to 3') of the sequence that was cloned as a promoter. The dotted line in the pSeS construct represents the 5' fragment that is absent.

\section{Figure 2: Promoter activity of the sequences upstream of the orf46 gene.}

GFP expression, measured as relative fluorescence intensity, in different insect cell lines infected with the different baculoviruses at a multiplicity of infection (MOI) of 5 . The fluorescence was measured at different time points after infection of Se301 (A), Sf21 (B), and Hi5 (C) cells. The results are expressed as the relative percentage of GFP fluorescence intensity, taken as $100 \%$ of the value corresponding to the maximum intensity obtained with the polh promoter. The values are the means of at least two independent assays. The error bars represent the standard error of the mean.

\section{Figure 3: Fluorescence microscopy of $\mathbf{S f 2 1}$ cells infected with the different baculoviruses.}

A representative image of Sf21 cells infected with a selected baculovirus at a MOI of 5. The images were taken at 48 hours post-infection. 
379 Figure 4: Promoter activity of pSeL120 when combined with the polh promoter.

380 GFP expression, measured as the relative fluorescence intensity, in different insect cell lines 381 infected with the different baculoviruses at a MOI of 5. The fluorescence was measured at 382 different time points after the infection of Se301 (A), Sf21 (B), and Hi5 (C) cells. The results are 383 expressed as the relative percentage of GFP fluorescence intensity, taken as $100 \%$ of the value 384 corresponding to the maximum intensity obtained with the polh promoter. The values are the 385 means of at least two independent assays. The error bars represent the standard error of the mean.

387 Figure 5: Promoter activity in insect larvae.

388 GFP expression, measured as relative fluorescence intensity, was obtained in insect larvae 389 infected with the different recombinant baculoviruses. The results are expressed as the relative 390 percentage of GFP fluorescence intensity, taken as $100 \%$ of the value corresponding to values 391 for the control sequence with the polh promoter. The values are the means of at least two 392 independent assays. The error bars represent the standard error of the mean. 
393

394

395

396

397

398

399

400

401

402

403

404

405

406

407

408

409

410

411

412

413

414

415

416

417

418

419

420

421

422

423

424

425

426

427

428

429

430

431

432

\section{References}

ChenY-R, Zhong S, Fei Z, Hashimoto Y, Xiang JZ, Zhang S, Blissard GW. 2013. "The Transcriptome of the Baculovirus Autographa Californica Multiple Nucleopolyhedrovirus in Trichoplusia Ni Cells." Journal of Virology 87 (11): 6391-6405. doi:10.1128/JVI.0019413.

Friesen PD 1997. "Regulation of Baculovirus Early Gene Expression.” In The Baculoviruses, 141-70. doi:10.1007/978-1-4899-1834-5_6.

Ge J, Jin L, Tang X, Gao D, An Q, Ping W. 2014. “Optimization of eGFP Expression Using a Modified Baculovirus Expression System.” Journal of Biotechnology 173 (1): 41-46. doi:10.1016/j.jbiotec.2014.01.003.

Gómez-Sebastián S, López-Vidal J, Escribano JM. 2014. "Significant Productivity Improvement of the Baculovirus Expression Vector System by Engineering a Novel Expression Cassette." Edited by Yi Li. PloS One 9 (5). Public Library of Science: e96562. doi:10.1371/journal.pone.0096562.

Gross CH, Rohrmann GF. 1993. "Analysis of the Role of 5' Promoter Elements and 3' Flanking Sequences on the Expression of a Baculovirus Polyhedron Envelope Protein Gene.” Virology. doi:10.1006/viro.1993.1030.

Gross CH, Russell RLQ, Rohrmann GF. 1994. “Orgyia Pseudotsugata Baculovirus p10 and Polyhedron Envelope Protein Genes: Analysis of Their Relative Expression Levels and Role in Polyhedron Structure." Journal of General Virology 75 (5): 1115-23. doi:10.1099/0022-1317-75-5-1115.

Hitchman RB, Locanto E, Possee RD, King LA. 2011. "Optimizing the Baculovirus Expression Vector System.” Methods 55 (1). Elsevier Inc.: 52-57. doi:10.1016/j.ymeth.2011.06.011.

Hitchman RB, Possee RD, Crombie AT, Chambers A, Ho K, Siaterli E, Lissina O, Sternard H, Novy R, Loomis K, Bird LE, Owens RJ, King LA. 2010. "Genetic Modification of a Baculovirus Vector for Increased Expression in Insect Cells." Cell Biology and Toxicology 26 (1): 57-68. doi:10.1007/s10565-009-9133-y.

Ishiyama, Seiji, and Masahiro Ikeda. 2010. "High-Level Expression and Improved Folding of Proteins by Using the vp39 Late Promoter Enhanced with Homologous DNA Regions." Biotechnology Letters 32 (11): 1637-47. doi:10.1007/s10529-010-0340-7.

Iwanaga M, Takaya K, Katsuma S, Ote M, Tanaka S, Kamita SG, Kang W, Shimada T, Kobayashi M. 2004. "Expression Profiling of Baculovirus Genes in Permissive and Nonpermissive Cell Lines." Biochemical and Biophysical Research Communications 323 (2): 599-614. doi:10.1016/j.bbrc.2004.08.114.

Jarvis DL. 2009. "Baculovirus-Insect Cell Expression Systems." Methods in Enzymology 463: 191-222. doi:10.1016/S0076-6879(09)63014-7.

Lee SY, Poloumienko A, Belfry S, Qu X, Chen W, MacAfee N, Morin B, Lucarotti C, Krause M. 1996. "A Common Pathway for p10 and Calyx Proteins in Progressive Stages of Polyhedron Envelope Assembly in AcMNPV-Infected Spodoptera Frugiperda Larvae." Archives of Virology, 2057-76. 
433

434

435

436

437

438

439

440

441

442

443

444

445

446

447

448

449

450

451

452

453

454

455

456

457

458

459

460

461

462

463

464

465

466

467

468

469

470

471

472

Li S, Wang H, Hu Z, Deng F. 2012. "Genetic Modification of Baculovirus Expression Vectors." Virologica Sinica 27 (2): 71-82. doi:10.1007/s12250-012-3236-y.

Lin C-H, Jarvis DL. 2012. "Utility of Temporally Distinct Baculovirus Promoters for Constitutive and Baculovirus-Inducible Transgene Expression in Transformed Insect Cells." Journal of Biotechnology 29 (6): 997-1003. doi:10.1016/j.biotechadv.2011.08.021.Secreted.

Lo HR, Chou CC, Wu TYY, Yuen JPY, Chao YC. 2002. "Novel Baculovirus DNA Elements Strongly Stimulate Activities of Exogenous and Endogenous Promoters." Journal of Biological Chemistry 277 (7): 5256-64. doi:10.1074/jbc.M108895200.

López-Vidal J, Gómez-Sebastián S, Sánchez-Ramos I, Escribano JM. 2013. "Characterization of a Trichoplusia Ni Hexamerin-Derived Promoter in the AcMNPV Baculovirus Vector." Journal of Biotechnology 165 (3-4). Elsevier B.V.: 201-8. doi:10.1016/j.jbiotec.2013.03.012.

Lu A, Miller LK. 1997. "Regulation of Baculovirus Late and Very Late Gene Expression." The Baculoviruses, Plenum, New York/London. 193-216.

Luckow VA, Summers MD. 1988. "Signals Important for High-Level Expression of Foreign Genes in Autographa Californica Nuclear Polyhedrosis Virus Expression Vectors." Virology 167 (1): 56-71. doi:10.1016/0042-6822(88)90054-2.

Manohar SL, Kanamasa S, Nishina T, Kato T, Park EY. 2010. "Enhanced Gene Expression in Insect Cells and Silkworm Larva by Modified Polyhedrin Promoter Using Repeated Burst Sequence and Very Late Transcriptional Factor-1." Biotechnology and Bioengineering 107 (6): 909-16. doi:10.1002/bit.22896.

Matsuura Y, Possee RD, Overton HA, Bishop DHL. 1987. "Baculovirus Expression Vectors : The Requirements for High Level Expression of Proteins , Including Glycoproteins." Journal of General Virology 68: 1233-50. doi:10.1099/0022-1317-68-5-1233.

Min MK, Bishop DHL. 1991. "Transcriptional Analyses of Baculovirus Polyhedrin and Foreign Gene Expression Relative to Baculovirus p10 mRNA Levels." The Journal of General Virology 72 ( Pt 10 (1991): 2551-56.

Morris TD, Miller LK. 1992. "Promoter Influence on Baculovirus-Mediated Gene Expression in Permissive and Nonpermissive Insect Cell Lines.” Journal of Virology 66 (12): 7397-7405.

O’Reilly DR, Miller LK, Luckow VA. 1994. Baculovirus Expression Vectors: A Laboratory Manual. Oxford University Press.

Ooi BG, Rankin C, Miller LK. 1989. "Downstream Sequences Augment Transcription from the Essential Initiation Site of a Baculovirus Polyhedrin Gene.” Journal of Molecular Biology 210 (4): 721-36.

Pascual L, Jakubowska AK, Blanca JM, Cañizares J, Ferré J, Gloeckner G, Vogel H, Herrero S. 2012. "The Transcriptome of Spodoptera Exigua Larvae Exposed to Different Types of Microbes.” Insect Biochemistry and Molecular Biology 42 (8): 557-70. doi:10.1016/j.ibmb.2012.04.003.

Rohrmann GF. 1999. "Nuclear Polyhedrosis Viruses.” In Encyclopedia of Virology, 2nd ed., 
473

474

475

476

477

478

479

480

481

482

483

484

485

486

487

488

489

490

491

492

493

494

495

496

497

498

499

500

501

502

503

504

505

506

507

508

146-52. Academic Press, London, United Kingdom.

Rohrmann GF. 2013. Baculovirus Molecular Biology. Third Edit. Bethesda (MD): National Center for Biotechnology Information (US).

Russell RL, Pearson MN, Rohrmann GF. 1991. "Immunoelectron Microscopic Examination of Orgyia Pseudotsugata Multicapsid Nuclear Polyhedrosis Virus-Infected Lymantria Dispar Cells: Time Course and Localization of Major Polyhedron-Associated Proteins." The Journal of General Virology 72 ( Pt 2) (2). Society for General Microbiology: 275-83. doi:10.1099/0022-1317-72-2-275.

Sano KI, Maeda K, Oki M, Maéda Y. 2002. "Enhancement of Protein Expression in Insect Cells by a Lobster Tropomyosin cDNA Leader Sequence.” FEBS Letters 532 (1-2): 143-46. doi:10.1016/S0014-5793(02)03659-1.

Smith GE, Summers MD, Fraser MJ. 1983. "Production of Human Beta Interferon in Insect Cells Infected with a Baculovirus Expression Vector." Molecular and Cellular Biology 3 (12): 2156-65. doi:10.1128/MCB.3.12.2156.Updated.

Thiem SM, Miller LK. 1990. "Differential Gene Expression Mediated by Late, Very Late and Hybrid Baculovirus Promoters.” Gene 91 (1): 87-94. doi:10.1016/0378-1119(90)90166-O.

Tiwari P, Saini S, Upmanyu S, Benjamin B, Tandon R, Saini KS, Sahdev S. 2010. "Enhanced Expression of Recombinant Proteins Utilizing a Modified Baculovirus Expression Vector." Molecular Biotechnology 46 (1): 80-89. doi:10.1007/s12033-010-9284-3.

van Lent JWM, Groenen JTM, Klinge-Roode EC, Rohrmann GF, Zuidema D, Vlak JM. 1990. "Localization of the $34 \mathrm{kDa}$ Polyhedron Envelope Protein in Spodoptera Frugiperda Cells Infected with Autographa Californica Nuclear Polyhedrosis Virus." Archives of Virology 111 (1-2): 103-14. doi:10.1007/BF01310508.

van Oers MM, Pijlman GP, Vlak JM. 2015. "Thirty Years of Baculovirus-Insect Cell Protein Expression: From Dark Horse to Mainstream Technology." Journal of General Virology 96: 6-23. doi:10.1099/vir.0.067108-0.

Venkaiah B, Viswanathan P, Habib S, Hasnain SE. 2004. "An Additional Copy of the Homologous Region (hr1) Sequence in the Autographa Californica Multinucleocapsid Polyhedrosis Virus Genome Promotes Hyperexpression of Foreign Genes.” Biochemistry 43 (25): 8143-51. doi:10.1021/bi049953q.

Wang R, Deng F, Hou D, Zhao Y, Guo L, Wang H, Hu Z. 2010. "Proteomics of the Autographa Californica Nucleopolyhedrovirus Budded Virions." Journal of Virology 84 (14): 7233-42. doi:10.1128/JVI.00040-10.

Weyer U, Possee RD. 1988. "Functional Analysis of the p10 Gene 5' Leader Sequence of the Autographa Californica Nuclear Polyhedrosis Virus." Nucleic Acids Research 16 (9): 3635-53. doi:10.1093/nar/16.9.3635. 


\section{Figure 1 (on next page)}

Schematic summary of the recombinant baculoviruses carrying different promoter regions employed in this study.

(A) Nucleotide sequence upstream of the orf46 gene from SeMNPV selected as a regulatory region (nucleotides 45417-47500 from AF169823). The arrows indicate the range of the fragments from the $5^{\prime}$ to $3^{\prime}$ sequence that were tested for promoter activity and the transcription initiation site $(+1)$. (B) Schematic representation of the recombinant baculoviruses generated which carry different fragments of the sequence upstream of the orf46 gene to test their promoter activity using GFP as a reporter. The white open arrows (pph) represent the polyhedrin promoter. The white boxes represent the GFP gene. The numbers indicate the first and the last nucleotides (from 5' to $3^{\prime}$ ) of the sequence that was cloned as a promoter. The dotted line in the pSeS construct represents the $5^{\prime}$ fragment that is absent. 


\section{Table $\mathbf{1}$ (on next page)}

ORFs from the SeMNPV highly expressed during infection of $S$. exigua larvae. (Pascual et al., 2012). 
1 Table 1. ORFs from the SeMNPV highly expressed during infection of S. exigua larvae.

2 (Pascual et al., 2012).

\begin{tabular}{lll}
\hline ORF & Description & Coverage \\
\hline ORF1 & Polyhedrin & 674 \\
\hline ORF46 & $\begin{array}{l}\text { Calyx/polyhedron } \\
\text { envelope protein }\end{array}$ & 590 \\
\hline ORF127 & lef6 & 516 \\
\hline ORF122 & - & 416 \\
\hline ORF94 & - & 347 \\
\hline ORF71 & odv-e25 & 344 \\
\hline ORF65 & $\begin{array}{l}\text { p6.9 DNA binding } \\
\text { protein }\end{array}$ & 262 \\
\hline ORF136 & odv-e18 & 255 \\
\hline ORF32 & pkip & 224 \\
\hline ORF124 & - & 204 \\
\hline
\end{tabular}

$3 \longdiv { { } ^ { 1 } \text { Coverage reported as the maximum coverage (number of reads) } }$ for a given ORF after mapping

4 of the SeMNPV genome with transcriptional data.

5

6 


\section{Figure 2 (on next page)}

Promoter activity of the sequences upstream of the orf46 gene.

GFP expression, measured as relative fluorescence intensity, in different insect cell lines infected with the different baculoviruses at a multiplicity of infection (MOI) of 5 . The fluorescence was measured at different time points after infection of Se301 (A), Sf21 (B), and $\mathrm{Hi5}$ (C) cells. The results are expressed as the relative percentage of GFP fluorescence intensity, taken as $100 \%$ of the value corresponding to the maximum intensity obtained with the pph promoter. The values are the means of at least two independent assays. The error bars represent the standard error of the mean. 

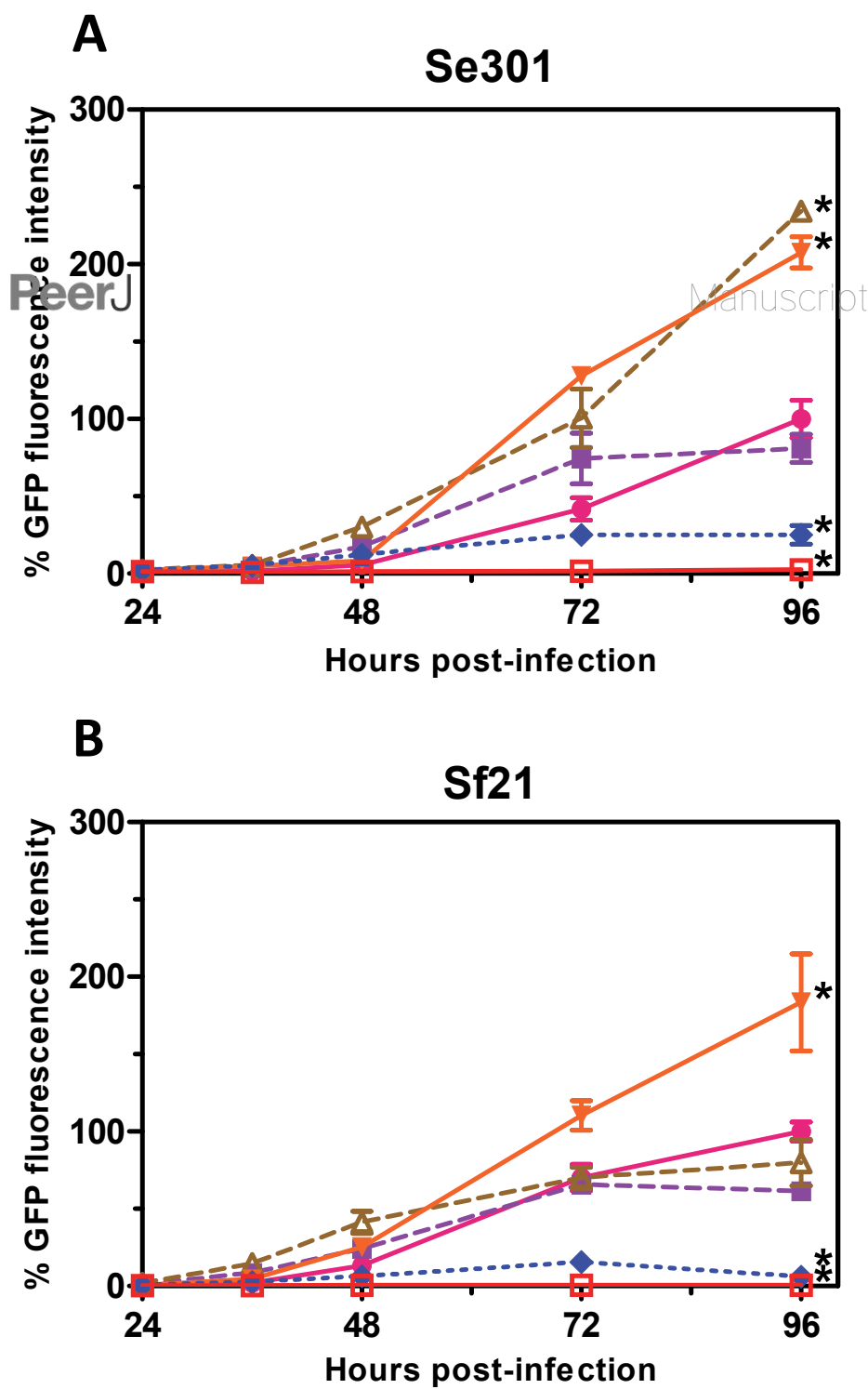
$\rightarrow$ polh
글 pSeL
-₫. pSeL140
- pSeL120
- p 131
$\bullet$ pSeS
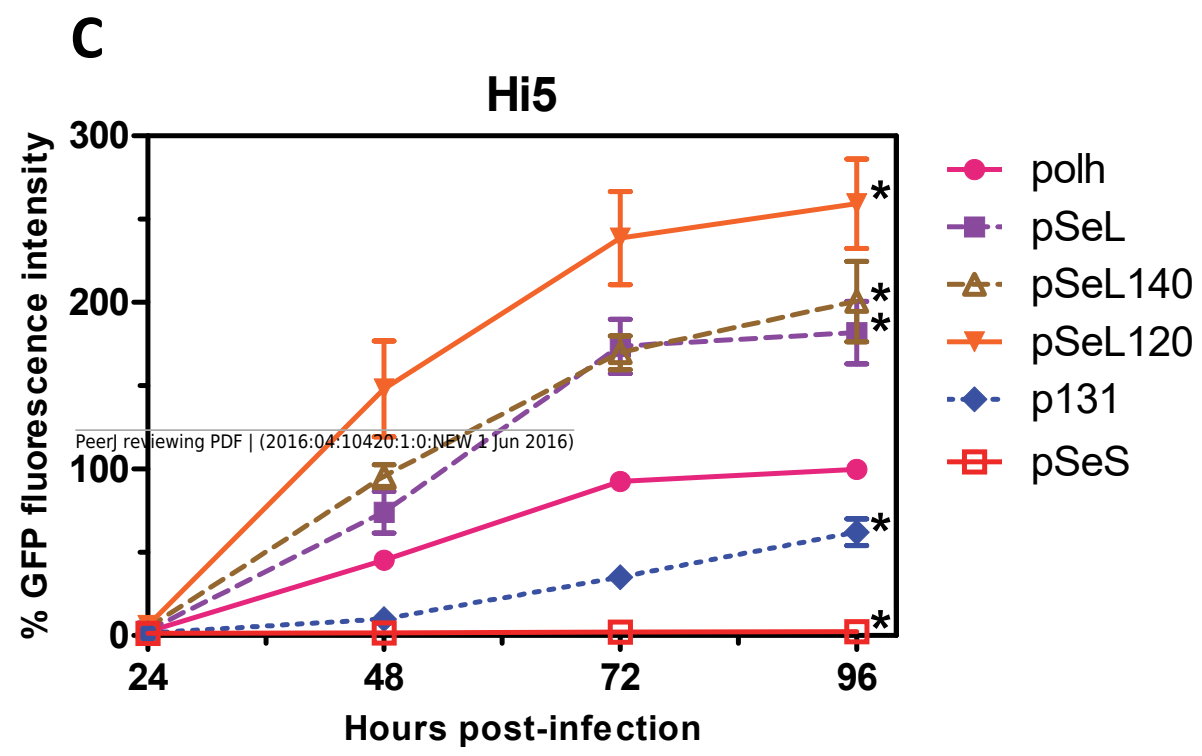
3

Fluorescence microscopy of Sf21 cells infected with the different baculoviruses.

A representative image of $\mathrm{Sf} 21$ cells infected with a selected baculovirus at a $\mathrm{MOI}$ of 5 . The images were taken at 48 hours post-infection. 
pSeS
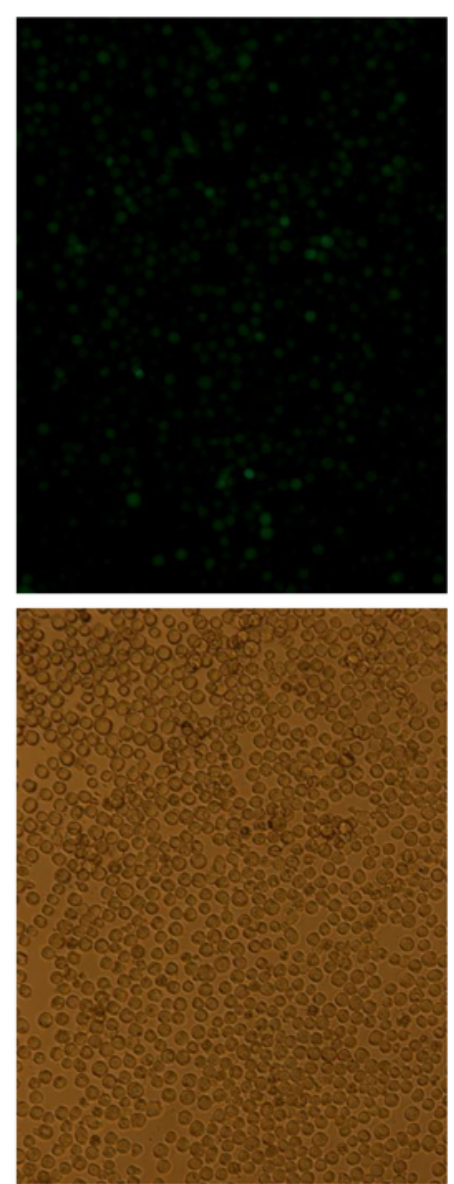

pph
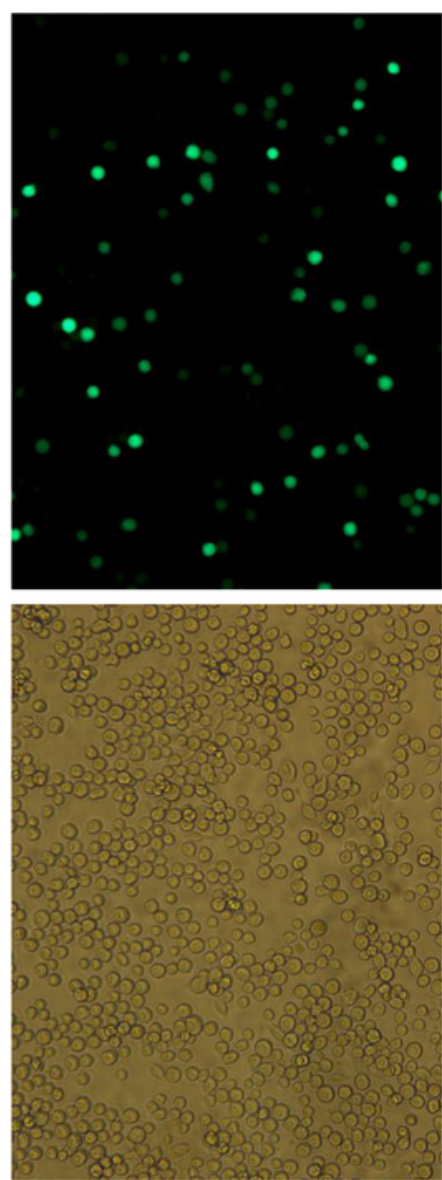

pSeL120
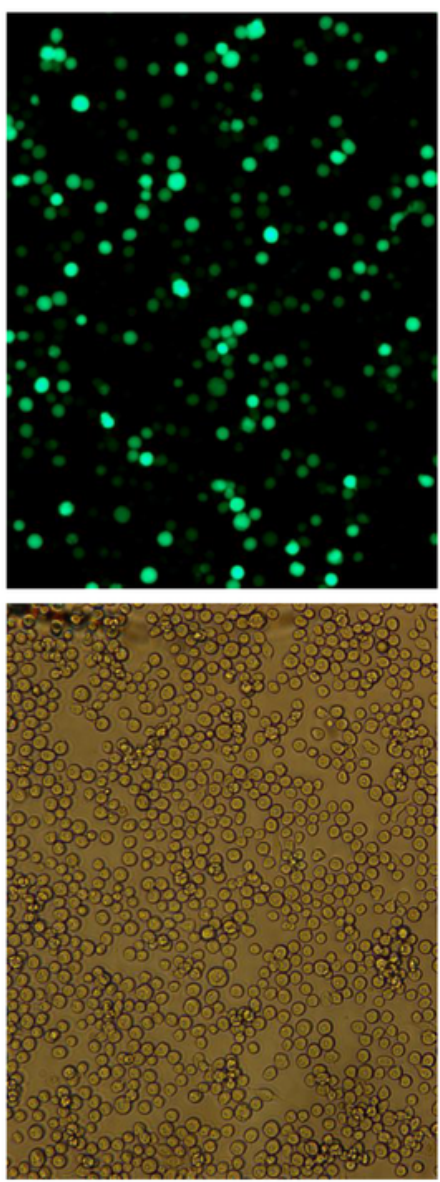

pph-pSeL
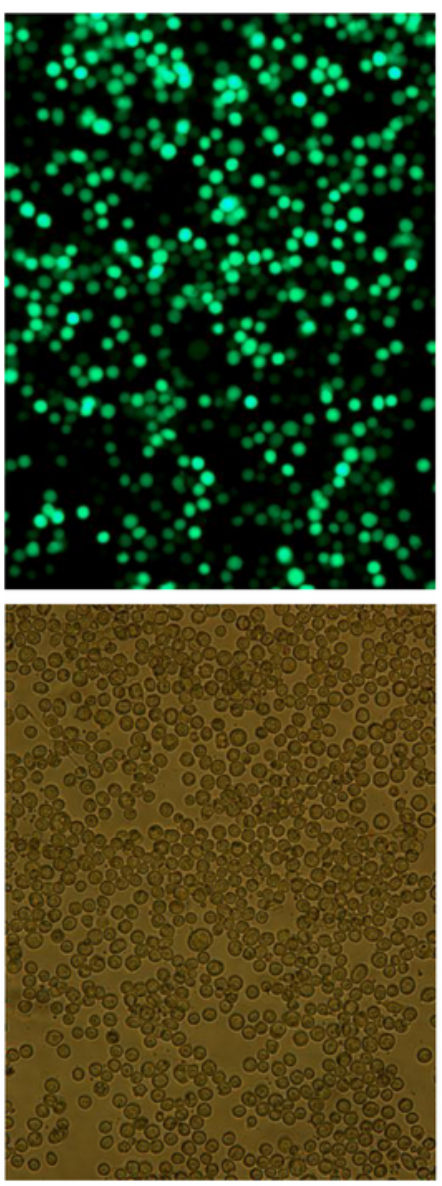
Figure 4(on next page)

Promoter activity of pSeL120 when combined with the pph promoter.

GFP expression, measured as the relative fluorescence intensity, in different insect cell lines infected with the different baculoviruses at a $\mathrm{MOI}$ of 5 . The fluorescence was measured at different time points after the infection of Se301 (A), Sf21 (B), and Hi5 (C) cells. The results are expressed as the relative percentage of GFP fluorescence intensity, taken as $100 \%$ of the value corresponding to the maximum intensity obtained with the pph promoter. The values are the means of at least two independent assays. The error bars represent the standard error of the mean. 


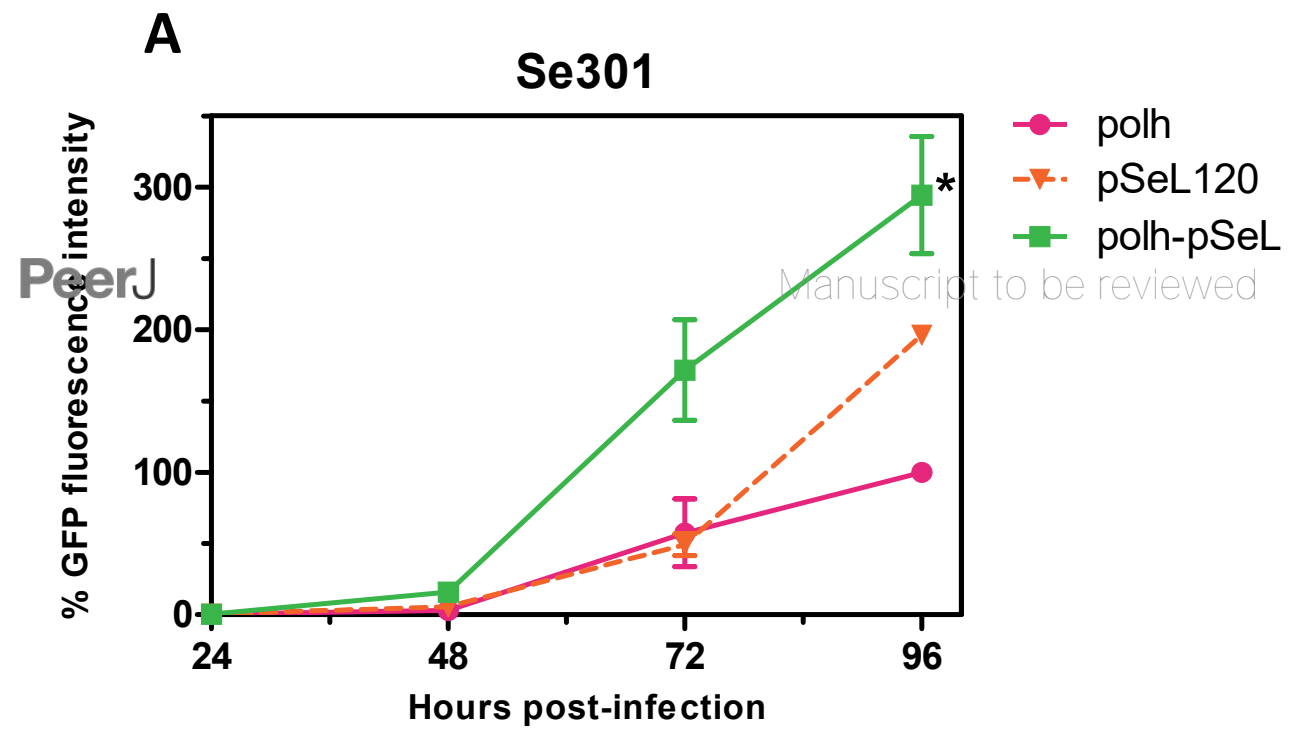

B

Sf21

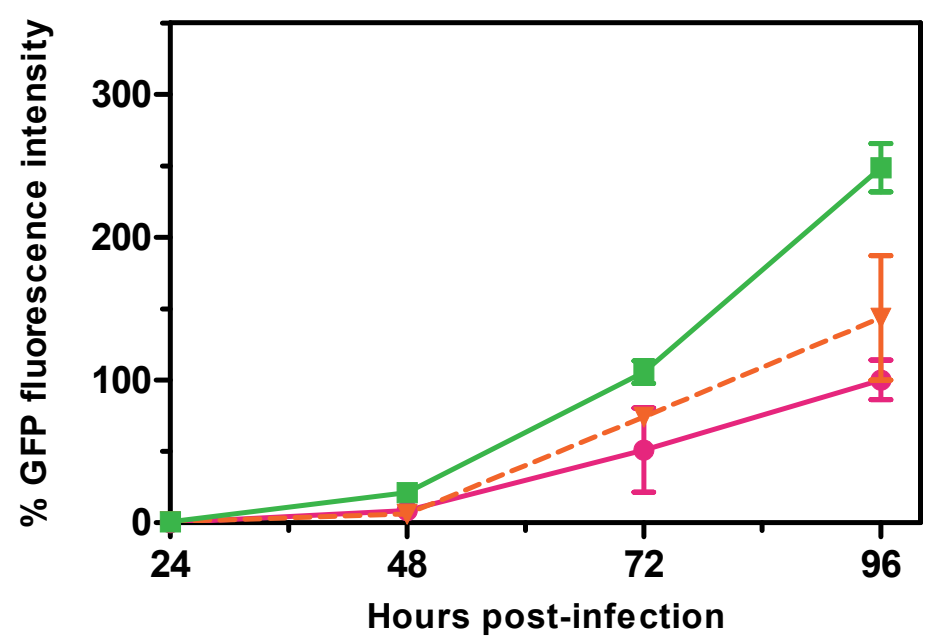

- - polh

-7. pSeL120

$\rightarrow$ polh-pSeL

C

Hi5

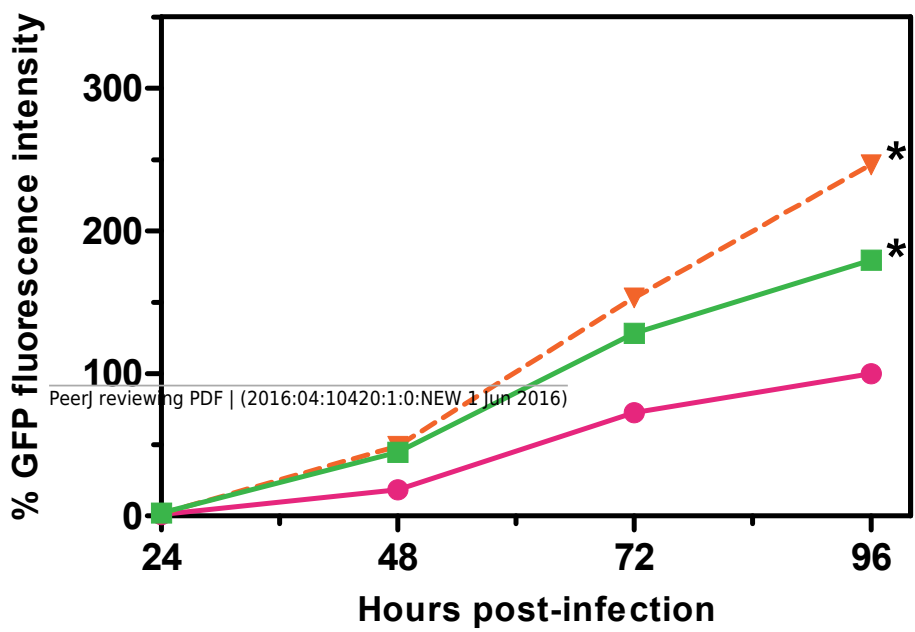

- - polh

-F. pSeL120

- polh-pSeL 
Figure $\mathbf{5}$ (on next page)

Promoter activity in insect larvae.

GFP expression, measured as relative fluorescence intensity, was obtained in insect larvae infected with the different recombinant baculoviruses. The results are expressed as the relative percentage of GFP fluorescence intensity, taken as $100 \%$ of the value corresponding to values for the control sequence with the pph promoter. The values are the means of at least two independent assays. The error bars represent the standard error of the mean. 
PeerJ A

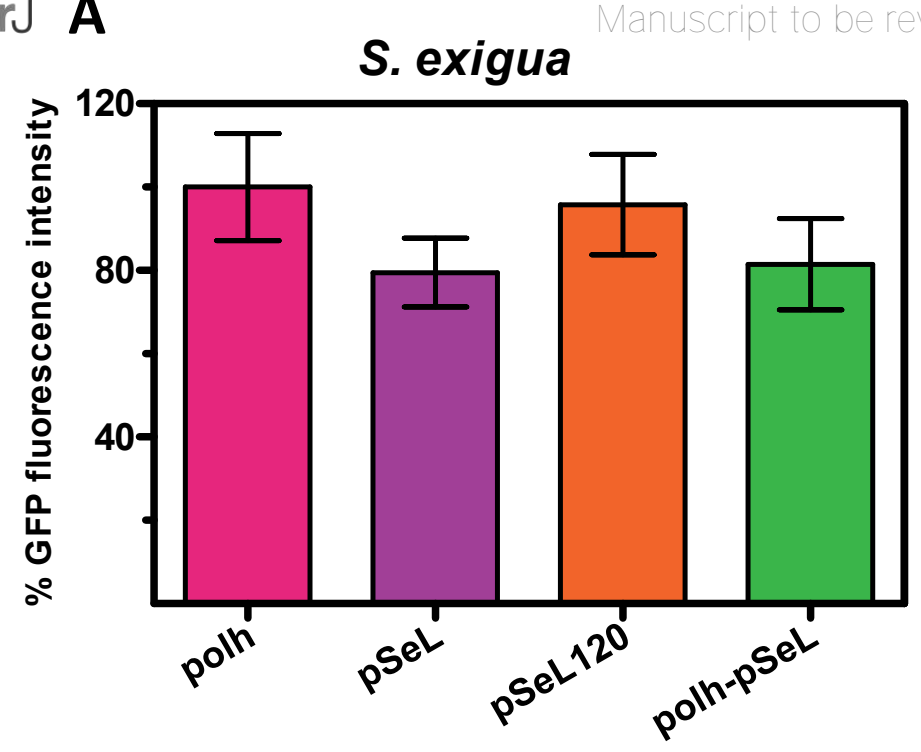

B

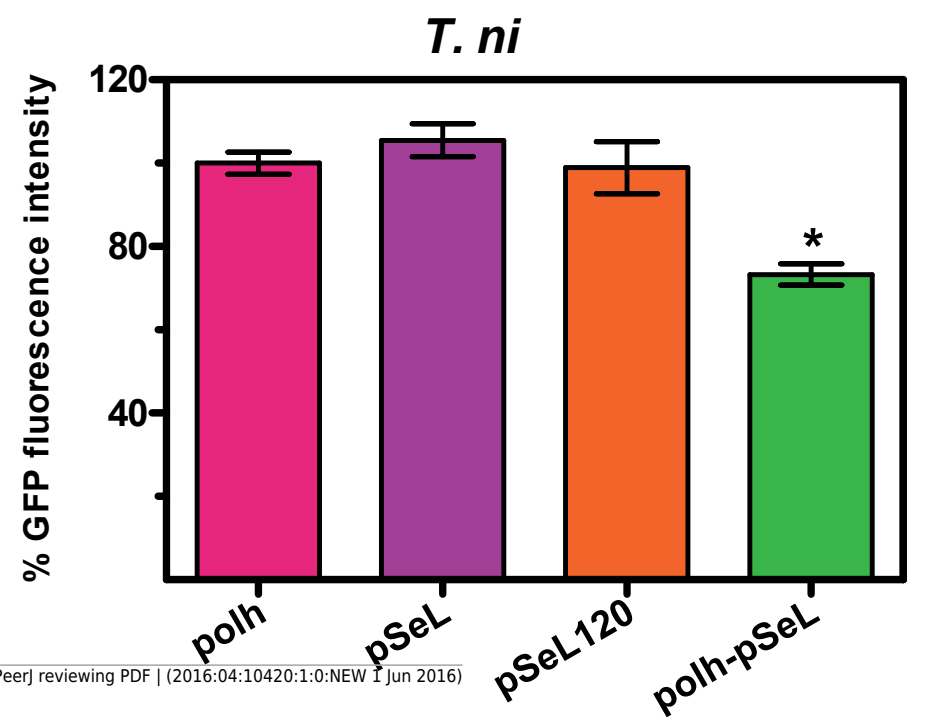

